\title{
Statistical analysis of electronic and phononic transport simulations of metallic atomic contacts
}

\author{
D. O. Möhrle $\odot,{ }^{1,2}$ F. Müller $\odot,{ }^{1}$ M. Matt, ${ }^{1}$ P. Nielaba, ${ }^{1}$ and F. Pauly $\oplus^{3,1}$ \\ ${ }^{1}$ Department of Physics, University of Konstanz, D-78457 Konstanz, Germany \\ ${ }^{2}$ German Aerospace Center, Pfaffenwaldring 38-40, D-70569 Stuttgart, Germany \\ ${ }^{3}$ Okinawa Institute of Science and Technology Graduate University, Onna-son, Okinawa 904-0495, Japan
}

(Received 19 July 2019; published 25 September 2019)

\begin{abstract}
We adapt existing phonon heat transport methods to compute the phononic thermal conductance of metallic atomic contacts during a stretching process. Nonequilibrium molecular dynamics simulations are used to generate atomic configurations and to simultaneously determine the phononic thermal conductance. Combining the approach with established electronic structure calculations based on a tight-binding parametrization allows us to calculate in addition charge transport properties of each contact geometry within the Landauer-Büttiker formalism. The method is computationally fast enough to perform a statistical analysis of many stretching events, and we apply it here to atomic junctions formed from three different metals, namely gold (Au), platinum (Pt), and aluminum (Al). The description of both phononic and electronic contributions to heat transport allows us to examine the validity of the Wiedemann-Franz law at the atomic scale. We find that it is well obeyed in the contact regime at room temperature for $\mathrm{Au}$ and $\mathrm{Al}$ as far as only electronic contributions are concerned, but deviations of up to $10 \%$ arise for Pt. If the total thermal conductance is studied, deviations of typically less than $10 \%$ arise for $\mathrm{Au}$ and $\mathrm{Al}$, which can be traced back mainly to phononic contributions to the thermal conductance, while electronic and phononic contributions can add up to some $20 \%$ for single-atom contacts of Pt.
\end{abstract}

DOI: $10.1103 /$ PhysRevB.100.125433

\section{INTRODUCTION}

The ongoing miniaturization of electronic devices promises an increase in speed and efficiency of operation. The physical limit of this miniaturization is given by integrated circuits consisting of functional units and wires made of single atoms. However, at the nanoscale heat dissipation becomes an increasingly important issue [1], and thermal management is of high relevance.

In this context, metallic atomic contacts serve as ideal systems to test charge and energy transport at the atomic scale [2,3]. Crucial progress in understanding thermopower [4,5], heat dissipation [6], and heat transport [7-9] in these systems has been made in recent years. The advances enabled tests of the Wiedemann-Franz law at the atomic scale, where transport is assumed to be phase coherent and elastic. In contrast the Wiedemann-Franz law, which connects electrical and thermal conductances, has been discovered empirically for macroscopic systems, where incoherent and inelastic scattering events also play a role. For metallic atomic contacts of $\mathrm{Au}$ and Pt only slight deviations from it were detected at room temperature [7].

Experimentally atomic contacts are realized using the scanning tunneling microscope (STM) technique [7,8,10,11], in which a STM tip is repeatedly dipped into a substrate and slowly separated again, or the mechanically controllable break junction method [12-14], which consists of a wire on a flexible sample that is bent until it narrows to cross sections of single atoms and finally breaks. A drawback of both methods is that in the contact regime, positions of individual atoms are not under precise control. For this reason different traces of electrical and thermal conductance versus distance appear, and data is analyzed statistically in terms of histograms to identify physical properties [2,7-9].

To understand the transport properties of atomic contacts, theoretical methods are typically applied to single junction geometries, which are assumed to correspond to an experimentally relevant geometry $[2,3]$. Theoretical work that generates a large amount of charge or energy transport data and analyzes it statistically is still scarce [5,15-21].

Recent theoretical work found the phononic contribution $\kappa_{\mathrm{ph}}$ to the total thermal conductance $\kappa$ to be small (less than $10 \%)$ in metallic atomic contacts of the heavy metals Au and Pt [21]. For the light Al, contributions of up to $20 \%$ were predicted due to the higher Debye energy $E_{\mathrm{D}}$ as compared to $\mathrm{Au}$ [21], which increases $\kappa_{\mathrm{ph}}$. For a particular single-atom junction geometry, the ratio $\kappa_{\mathrm{ph}} / \kappa$ has even been computed to reach as much as $40 \%$ [21], which was mainly due to a suppression of the electronic transmission and hence the electronic thermal conductance $\kappa_{\mathrm{el}}$.

Here we extend our previous work [21] in order to study electronic and phononic contributions to the thermal conductance for a statistically significant set of atomic junction geometries. For this purpose we combine a nonequilibrium molecular dynamics (NEMD) approach for the determination of junction geometries and of phonon heat transport properties with a tight-binding method for the electronic structure description and subsequent electronic transport calculations $[22,23]$. As compared to previous molecular-dynamics-based work $[15,16]$, we are thus adding here the description of $\kappa_{\mathrm{ph}}$. The combined approach is computationally fast enough to enable the study of a larger number of junction stretching processes. In this way, we are not only able to analyze histograms of the electrical conductance and 
electronic thermal conductance but also of the phononic heat conductance.

We choose the metals $\mathrm{Au}, \mathrm{Pt}$, and $\mathrm{Al}$ due to their different properties. It is well known by now that the electrical conductance $G$ is quantized in atomically thin gold nanocontacts [24,25], as a result of $s$ orbitals being the main contributors to the transmission $[14,15]$. For platinum, the $d$ orbitals also need to be considered, leading to a larger conductance than for gold for similar atomic configurations and to no conductance quantization $[12,16]$. In aluminum, the $p$ orbitals show contributions in addition to the $s$ orbitals [26,27]. The electrical transport properties are by now well understood in contrast to the thermal transport properties, which became experimentally accessible at room temperature only recently [7-9]. On the side of heat transport, the different electronic properties of $\mathrm{Au}, \mathrm{Pt}$, and $\mathrm{Al}$ are expected to be reflected in the electronic heat conductance. Concerning phonon heat transport the choice of the metals is also interesting, since the Debye energies of $\mathrm{Au}$ and $\mathrm{Pt}$ of 15 and $20 \mathrm{meV}$, respectively, are very similar as well as their atomic mass, while that of Al of $34 \mathrm{meV}$ is nearly double the size and the atoms are lighter by a factor of more than 7 [28].

In nanojunctions heat transport via photons may yield another contribution, beside those of electrons and phonons discussed here. Indeed evanescent waves can enhance radiative thermal transport contributions in nanogaps, as studied in recent experiments [29-31]. Since we focus in this work on the contact regime and the precise magnitude depends on assumptions about the macroscopic shape of the electrodes [32], we will neglect radiative heat transport. Estimates yield some $10 \mathrm{pW} / \mathrm{K}$ for the photonic thermal conductance [7,32], which is below the phononic thermal conductance values obtained here and may amount to some $1 \%$ of $\kappa$ for typical junction geometries.

The paper is organized as follows. In Sec. II the theoretical models used to generate junction geometries and to describe phonon and electron heat transport are presented together with the Wiedemann-Franz law. Section III discusses the results. For each of the three materials, $\mathrm{Au}, \mathrm{Pt}$, and $\mathrm{Al}$, we show a single stretching process first, followed by a statistical analysis of properties collected from 60 such processes. Finally, we summarize our conclusions in Sec. IV. Some technical details, namely a full junction stretching process and the computational costs of our simulations, are presented in Sec. A of the Appendix.

\section{THEORETICAL MODEL}

We use NEMD simulations to determine atomic configurations and phononic thermal conductance values. Utilizing a tight-binding model, we compute the electronic structure of each junction geometry and derive electronic transport properties from it within the Landauer-Büttiker formalism, namely the electrical conductance and the electronic thermal conductance. We describe the details of these procedures in the following.

\section{A. Junction geometries}

To determine junction geometries, we use NEMD simulations and the program package LAMMPS [33,34]. The forces between atoms are parametrized by the embeddedatom method (EAM) [35], and the potentials are taken from Ref. [36].

The initial metallic atomic junction configurations are shown in Fig. 1. These nanowires with a squarelike cross section are oriented along the (100) crystallographic direction and consist of 1126 atoms in total. We have selected the (100) direction, because studies of gold nanowires with a transmission electron microscope have revealed that just before rupture they are crystalline and display only atomic configurations, where (100), (110), or (111) directions lie parallel to the stretching direction [37]. The 121 large red and 121 large blue spheres denote atoms of the hot and cold reservoirs for phonon heat transport simulations, respectively. The thermal reservoirs are connected via 226 flexible wire atoms, represented by the large gray spheres. The five atomic layers of the wire are narrowing down stepwise towards the center. Small gray atoms surrounding the reservoirs have fixed positions and interact with other atoms via their potential. As depicted in Fig. 1, the reservoirs and surrounding fixed

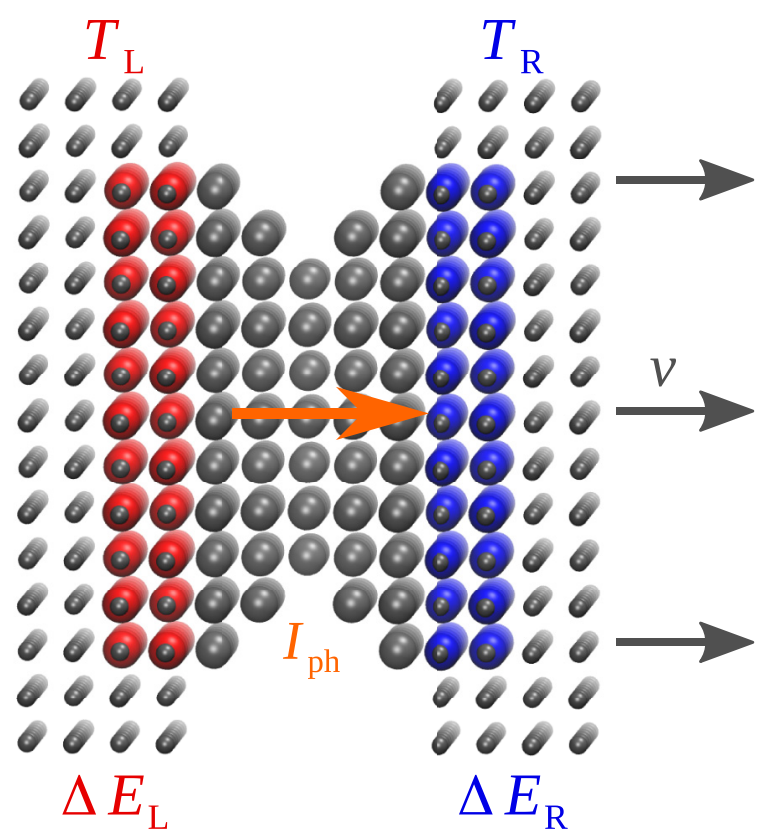

FIG. 1. Starting configuration for the NEMD simulations. The transport in the square-shaped nanowire is oriented along the (100) crystallographic direction. Interatomic potentials and lattice parameters are adjusted for the different metals. Two thermostats are applied to the red and blue reservoir atoms on the left $(\mathrm{L})$ and right $(\mathrm{R})$ side at actual temperatures $T_{\mathrm{L}}$ and $T_{\mathrm{R}}$. The energy changes $\Delta E_{\mathrm{L}}$ and $\Delta E_{\mathrm{R}}$ due to the thermostats are related to the heat transported by the phononic current $I_{\mathrm{ph}}$, visualized by the orange arrow in the middle of the nanowire. Small atoms surrounding the thermal reservoir atoms are held fixed. During the simulation, the "fixed" atoms on the right change their positions at a constant velocity $v$, as indicated by three arrows. The large gray atoms between the thermal reservoirs are the wire atoms, whose motion is free of external constraints. We note that when trying to estimate the number of atoms in the junction from the picture, the face-centered cubic lattice geometry needs to be taken into account. In the side view shown not all of the atoms that appear to be in a front row are actually located in the same plane. 
atom groups are two atomic layers deep. The purpose of the fixed atoms, totaling a number of $2 \times 329$, is to stabilize the geometries of the thermal reservoirs and in this way mimic the behavior of two semi-infinite crystalline electrodes. We have varied the size of the thermal reservoirs and found no significant changes in the resulting phononic thermal transport. Relatively small reservoir sizes are thus chosen in order to decrease computation times, especially for the electronic structure calculations to be described later.

The equations of motion of the wire and reservoir atoms are solved using the Velocity-Verlet integration method with a time step of $1 \mathrm{fs}$. Each of the two reservoirs is kept at a nearly constant temperature of $T_{\mathrm{L}}=330 \mathrm{~K}$ and $T_{\mathrm{R}}=300 \mathrm{~K}$ by a separate velocity rescale thermostat. The velocity rescale thermostats, as implemented in LAMMPS, are used because they provide a stable temperature for different reservoir and wire sizes and are computationally efficient.

To simulate a junction stretching event, we start as follows. In the thermal reservoirs and the wire part velocities of the atoms are selected at random in such a way that a Gaussian distribution centered at $300 \mathrm{~K}$ is achieved. Accordingly both thermostats are set to a temperature of $300 \mathrm{~K}$. After an equilibration time of $1 \mathrm{~ns}$ thermostat $\mathrm{L}$ slowly increases the temperature of its reservoir from 300 to $330 \mathrm{~K}$ over a duration of $1 \mathrm{~ns}$, while thermostat $\mathrm{R}$ remains at the temperature of $300 \mathrm{~K}$. After an additional equilibration time of $1 \mathrm{~ns}$ the stretching process begins by separating the group of fixed atoms surrounding the cold reservoir $\mathrm{R}$ at a constant velocity of $v=0.01 \mathrm{~m} / \mathrm{s}$ from the hot reservoir L. This leads to a narrowing of the wire and a typical conductance-displacement trace comparable to those observed in experimental setups.

\section{B. Phonon transport}

The thermostats used in the NEMD simulation rescale the velocities of the $N_{X}$ atoms considered in reservoir $X=\mathrm{L}, \mathrm{R}$, if the difference between the actual and the desired temperature according to the equipartition theorem is greater than the predefined window setting of the thermostat:

$$
\left\langle E_{X}\right\rangle=\left\langle\sum_{i=1}^{N_{X}} \frac{m_{i}}{2} v_{i}^{2}\right\rangle=\frac{3}{2} N_{X} k_{\mathrm{B}} T_{X} .
$$

In the expression $E_{X}$ and $T_{X}$ are the actual energy and temperature of the reservoir $X, v_{i}$ and $m_{i}$ are the velocity and mass of the $i$ th particle, and $k_{\mathrm{B}}$ is the Boltzmann constant. The rescaling leads to an energy change,

$$
\Delta E_{X}=\alpha\left\langle E_{X}\right\rangle\left(1-\frac{T_{\mathrm{th}, \mathrm{X}}}{T_{X}}\right),
$$

where $T_{X}$ is the temperature of the system before the rescaling and $T_{\mathrm{th}, X}$ is the target temperature of the thermostat. Due to the fraction factor $\alpha$, the thermostat does not rescale fully to the desired temperature. (We choose the settings of the thermostat as window $=0.01 \mathrm{~K}$ and fraction $\alpha=0.8$ to allow small fluctuations of the temperature.) Note that the sign of the energy change in Eq. (2) is positive, if the system needs to be cooled, and negative if it needs to be heated. This choice of sign is consistent with the data output of the thermostats in LAMMPS.
As visible in Fig. 1, the energy difference $-\Delta E_{\mathrm{L}}$ is supplied to the hot reservoir by thermostat $\mathrm{L}$ and $-\Delta E_{\mathrm{R}}$ to the cold reservoir by thermostat $\mathrm{R}$ during each time step. The orange arrow indicates the phononic heat current $I_{\mathrm{ph}}$, which transports energy from the hot $(\mathrm{L}, \mathrm{red})$ to the cold $(\mathrm{R}$, blue) reservoir. We can thus determine the phononic thermal conductance using Fourier's law via

$$
\kappa_{\mathrm{ph}}=\frac{I_{\mathrm{ph}}}{T_{\mathrm{L}}-T_{\mathrm{R}}}=\frac{1}{2 \Delta t} \frac{-\Delta E_{\mathrm{L}}+\Delta E_{\mathrm{R}}}{T_{\mathrm{L}}-T_{\mathrm{R}}} .
$$

We store the energy changes $\Delta E_{\mathrm{L}}, \Delta E_{\mathrm{R}}$ and temperatures $T_{\mathrm{L}}, T_{\mathrm{R}}$ every 1000 simulation time steps, corresponding to $\Delta t=1 \mathrm{ps}$. Since the fluctuations of $\Delta E_{\mathrm{L}}$ and $\Delta E_{\mathrm{R}}$ are large, we smoothed $\Delta E_{\mathrm{L}}, \Delta E_{\mathrm{R}}, T_{\mathrm{L}}, T_{\mathrm{R}}$ by using a moving average with a Gaussian weight function, cut off at each side at the standard deviation of $1 \mathrm{~ns}$. From this data we compute $\kappa_{\mathrm{ph}}$ via Eq. (3). In order to compress the data to the 1-ns time step used in the electronic transport calculations to be described next, we performed an arithmetic average over $1 \mathrm{~ns}$ around the time point of the prospective electronic transport calculation to obtain the final value for $\kappa_{\mathrm{ph}}$. Due to the classical NEMD simulations we expect that studies of phonon heat flow with the present technique are limited to temperatures above $100 \mathrm{~K}$.

\section{Electron transport}

To calculate the electronic transport properties, we use the Landauer-Büttiker scheme. For a detailed description we refer to Refs. [3,15,16,21].

In the linear-response regime the Landauer-Büttiker formalism states that the electrical conductance is given by

$$
G=G_{0} K_{0},
$$

and the electronic thermal conductance by

$$
\kappa_{\mathrm{el}}=\frac{2}{h T}\left(K_{2}-\frac{K_{1}^{2}}{K_{0}}\right)
$$

with

$$
K_{n}=\int d E(E-\mu)^{n} \tau(E)\left(\frac{-\partial f(E, T)}{\partial E}\right),
$$

the conductance quantum $G_{0}=2 e^{2} / h$, the electronic transmission $\tau(E)$, and the Fermi function $f(E, T)$. In our calculations, we will set the electronic temperature to the average of the target temperatures of the two thermostats, $T=315 \mathrm{~K}$.

We need to have information about the electronic structure in order to compute the electronic transmission $\tau(E)$. For this purpose we employ a tight-binding model [22,23], which parametrizes the Hamiltonian through the Slater-Koster approximation [38]. Since the bulk behavior differs from those at surfaces or in nanowires, we modified the approach to ensure self-consistently a local charge neutrality of the wire atoms, as explained in previous work $[15,16]$.

For the calculation of the electronic transmission and hence $G$ and $\kappa_{\mathrm{el}}$ we redefine the central "wire" part as compared to the phonon heat transport calculations discussed before. For every geometric configuration the central part is chosen to consist of the two thermal reservoirs (red and blue large spheres) as well as the large gray wire atoms, totaling 468 atoms. The two outer layers of the fixed atoms on both 
sides (small gray spheres in Fig. 1, 225 atoms on each side) are assumed to belong to semi-infinite, perfect face-centered cubic electrode crystals. The 104 fixed atoms on each side, surrounding the colored phonon thermal reservoirs perpendicular to the heat flow, are ignored for the electronic calculations to shorten computation times.

\section{Wiedemann-Franz law}

The empirical Wiedemann-Franz law connects the electrical conductance $G$ with the electronic thermal conductance $\kappa_{\text {el }}$ via

$$
\kappa_{\mathrm{el}}=L_{0} T G .
$$

Here the Lorenz number is given by $L_{0}=\left(\pi^{2} / 3\right)\left(k_{\mathrm{B}} / e\right)^{2}$ with the elementary electronic charge $e=|e|$.

Equation (7) allows one to define the quantum of thermal conductance as $\kappa_{0}=L_{0} T G_{0}$. If we use $\kappa_{0}$ in the following, we will set the absolute temperature to $T=315 \mathrm{~K}$, consistently with our choice in Sec. IIC.

Subsequently we will use the electronic Lorenz ratio,

$$
\frac{L_{\mathrm{el}}}{L_{0}}=\frac{\kappa_{\mathrm{el}}}{L_{0} T G},
$$

to discuss the validity of the Wiedemann-Franz law for our simulations. A value of $L_{\mathrm{el}} / L_{0}=1$ indicates perfect agreement with it and considers only electronic transport quantities $G$ and $\kappa_{\mathrm{el}}$. Since in the experiments only the total thermal conductance $\kappa=\kappa_{\mathrm{el}}+\kappa_{\mathrm{ph}}$ can be measured, we also introduce the Lorenz ratio,

$$
\frac{L}{L_{0}}=\frac{\kappa}{L_{0} T G}
$$

to quantify deviations from a "generalized" WiedemannFranz law $\kappa=L_{0} T G$ due to both electrons and phonons.

\section{RESULTS AND DISCUSSION}

In this section we present the simulation results for the three materials $\mathrm{Au}, \mathrm{Pt}$, and $\mathrm{Al}$. We performed 60 stretching events for atomic junctions of each metal. Different junction evolutions were obtained by selecting random initial velocities of wire and thermal reservoir atoms. After discussing exemplarily a single stretching process, we will analyze the 60 junction evolutions and related transport properties statistically.

\section{A. Gold}

\section{Single stretching process}

Figure 2 shows a single stretching process for a gold nanojunction. Figure 2(a) presents the sum of all forces acting in the direction of the stretching on the fixed atoms that surround the cold reservoir. Plastic and elastic stages can be distinguished. During the elastic stages forces build up, which decrease in plastic stages, when atomic bonds break and atoms in the junction rearrange. The conductance $G$ (blue squares), electronic thermal conductance $\kappa_{\mathrm{el}}$ (red crosses), and the tenfold magnified phononic thermal conductance $\kappa_{\mathrm{ph}}$ (orange dots) versus electrode displacement are depicted in Fig. 2(b). The electrical conductance exhibits a trace with
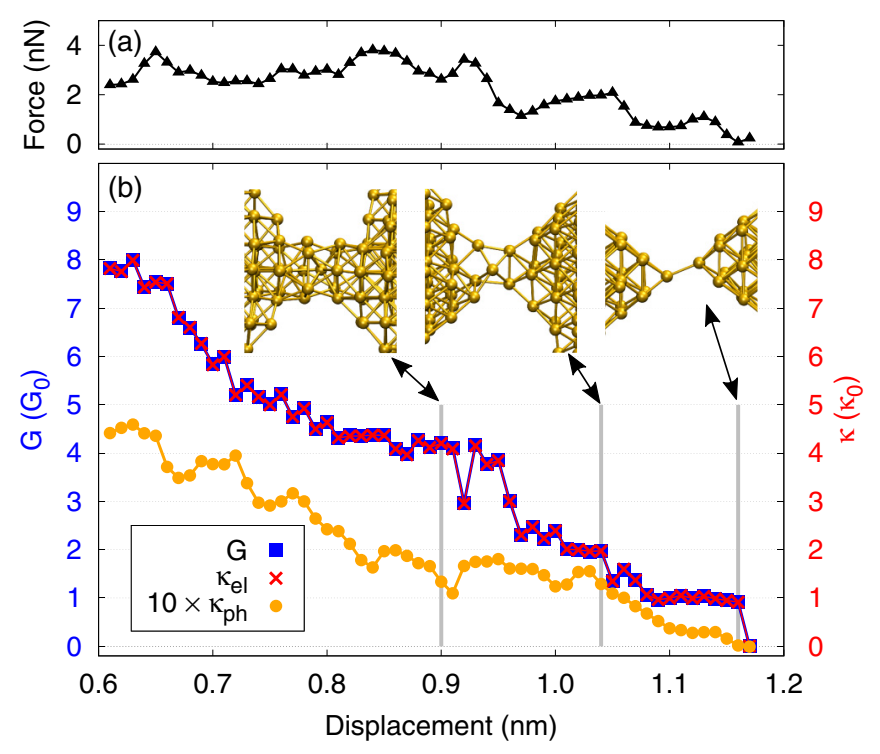

FIG. 2. Single stretching process for Au. (a) Force acting on the fixed atoms of the right side (see Fig. 1) as a function of electrode displacement. (b) Electrical conductance $G$ (blue squares) and electronic as well as phononic thermal conductance $\kappa_{\mathrm{el}}$ (red crosses) and $\kappa_{\mathrm{ph}}$ (orange circles) as a function of electrode displacement. Note that the phononic thermal conductance is magnified by a factor of 10 . The three insets show the narrowest part of the wire at displacements of $0.90 \mathrm{~nm}, 1.04 \mathrm{~nm}$, and $1.16 \mathrm{~nm}$, as indicated by vertical gray lines.

typical features seen in experimental results [24,25]. Before junction rupture at a displacement of $1.16 \mathrm{~nm}$, a plateau at $1 G_{0}$ can be observed. This plateau is a result of the atomic configuration shown as an inset on the right. This dimer configuration consists basically of a two-atom chain formed from the tip atoms of two atomically sharp electrode pyramids that touch each other. Before this last plateau, a shorter plateau at roughly $2 G_{0}$ is found. The middle inset shows the corresponding atomic configuration. At an even smaller electrode separation, we see a plateau at a conductance of $4 G_{0}$. The left inset relates this to a structure with two pentagonal rings and a single atom between them.

Measuring $G$ and $\kappa_{\mathrm{el}}$ in terms of their respective conductance quanta, we find a nearly perfect match between both quantities throughout the whole stretching process. This is expected from the Wiedemann-Franz law in Eq. (7), if the transmission function $\tau(E)$ is weakly energy dependent around the Fermi energy, as it is typically the case for $\mathrm{Au}$ atomic contacts $[21,39]$.

The phononic thermal conductance $\kappa_{\mathrm{ph}}$ decreases overall within the simulation run. For structures with large narrowest cross sections at small displacements at the beginning of the simulations (see the plot and discussion in Sec. A), it shows a clear saw-tooth-like profile. During elastic displacements of atoms, the force increases steadily, while the phononic thermal conductance tends to decrease. For a displacement large enough, the structure then reconfigures, reducing the force and abruptly increasing the phononic thermal conductance. This can be understood by considering the force constants, which are changing with interatomic distance for anharmonic potentials. The decreasing force constants lead to a decrease 
in the phononic thermal conductance until they are restored again after the reconfiguration [21]. For the rather elongated structures shown in Fig. 2 this behavior is, however, not very pronounced.

Between a displacement of $1.0 \mathrm{~nm}$ and the breaking of the wire at $1.16 \mathrm{~nm}$, two steps in the force trace can be observed. The first step is accompanied by a reconfiguration of the structure with a conductance of $2 G_{0}$ to the dimer structure with a conductance of $1 G_{0} . \kappa_{\mathrm{ph}}$ follows this decline in the force more smoothly than $\kappa_{\mathrm{el}}$. The second step in the force is due to the breaking of the wire. While the electronic transport properties drop to zero from a plateau at $1 G_{0}$ or $1 \kappa_{0}$, respectively, $\kappa_{\mathrm{ph}}$ decreases rather smoothly. We note that the behavior of $\kappa_{\mathrm{ph}}$ in this case resembles closely the force, which shows a rather continuous decrease before the point of rupture in contrast to the sharp drop of the electronic transport properties. This can be understood by recalling that in the case of a dimer contact the force and $\kappa_{\mathrm{ph}}$ will basically be determined by the mechanical interaction between the two tip atoms.

The study of Fig. 2 reveals that $\kappa_{\text {ph }}$ reacts somewhat differently to certain atomic reconfigurations than $\kappa_{\mathrm{el}}$ (or, equivalently, $G$ ), as discussed in the previous paragraph. Another example can be seen in the range of displacements between 0.80 and $1.04 \mathrm{~nm}$. Here the electronic thermal conductance $\kappa_{\mathrm{el}}$ stays roughly constant at a value of around $4.5 \kappa_{0}$ and then drops rapidly towards $2 \kappa_{0}$, with the exception of the dip at $0.92 \mathrm{~nm}$. In contrast $\kappa_{\mathrm{ph}}$ shows two smaller dips but then remains roughly constant during the rapid drop of $\kappa_{\mathrm{el}}$. Fundamentally this behavior originates from differences in the sensitivity of mechanical interatomic interactions and electronic structure to atomic junction configurations.

\section{Statistical analysis}

Let us now take a look at the results of 60 stretching processes, each of them similar to the simulation run described in the previous paragraph. We will analyze these results statistically in the following.

Figure 3(a) shows the histogram of the electrical conductance $G$, Fig. 3(b) those of the total thermal conductance $\kappa$, and in the inset those of the phononic thermal conductance $\kappa_{\text {ph }}$. The histogram for $G$ exhibits a large peak at $1 G_{0}$, which corresponds to the plateau at $1 G_{0}$ for the single conductancedistance trace in Fig. 2(b) and is in agreement with experimental results $[7,24,25]$. A small peak at $2 G_{0}$ is hardly discernible. Instead it is more likely in our simulations to find configurations with conductances centered around $2.7 G_{0}$. Another area of increased counts is visible at $4.5 G_{0}$. It stems mainly from the structures with two pentagonal rings interconnected by a single atom, discussed in Fig. 2.

The thermal conductance histogram for $\kappa$ closely resembles those of the electrical conductance $G$. This can be anticipated from the single stretching trace, which manifests near perfect proportionality between $G$ and $\kappa_{\mathrm{el}}$, as expected from the Wiedemann-Franz law in Eq. (7), and only a minor contribution of $\kappa_{\mathrm{ph}}$. Figure 2 indeed shows that $\kappa_{\mathrm{ph}}$ is more than an order of magnitude smaller than $\kappa_{\mathrm{el}}$. In detail we find that peaks in the thermal conductance histogram are shifted slightly towards larger values than for $G$, which we attribute basically to the phononic contribution.
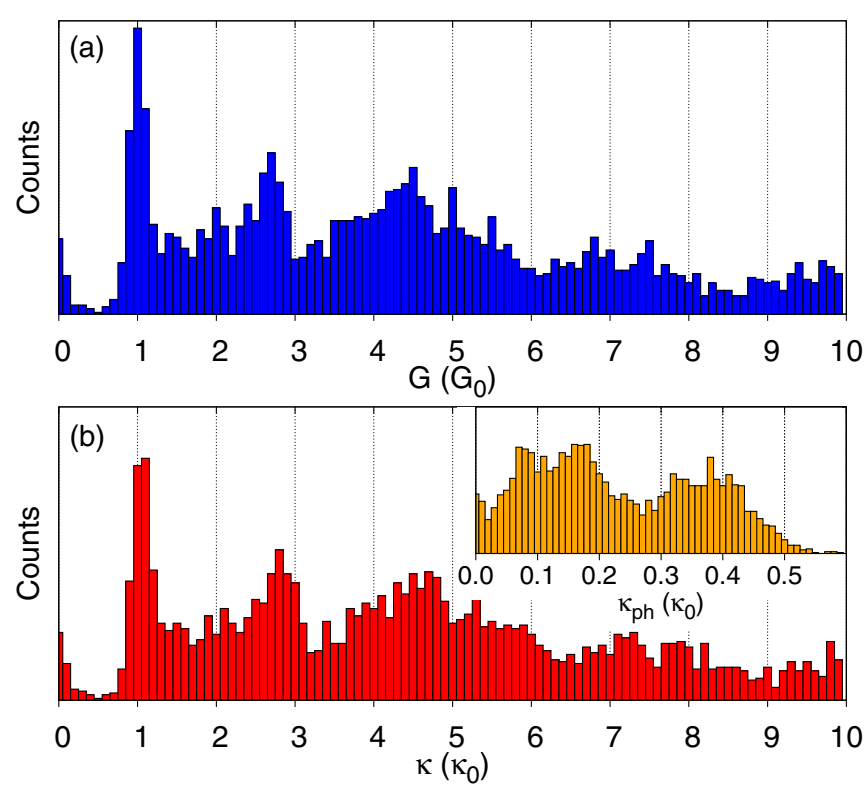

FIG. 3. Histograms for Au for (a) the electrical conductance $G$, (b) the total thermal conductance $\kappa=\kappa_{\mathrm{el}}+\kappa_{\mathrm{ph}}$, and in the inset for $\kappa_{\mathrm{ph}}$. We use bin sizes of $0.1 G_{0}$ and $0.1 \kappa_{0}$ for electrical and thermal conductances, respectively.

The phononic thermal conductance $\kappa_{\mathrm{ph}}$ in the inset of Fig. 3(b) shows no clear peaks. Instead there are two broad intervals of high counts centered around $0.13 \kappa_{0}$ and $0.37 \kappa_{0}$. The latter region results from junctions at the beginning of the pulling process shown in Fig. 2. The area around $0.13 \kappa_{0}$, on the other hand, originates from a nearly constant $\kappa_{\text {ph }}$ while $G$ changes from more than $4 G_{0}$ to less than $2 G_{0}$, seen again in Fig. 2 . The counts at values smaller than $0.05 \kappa_{0}$ stem mainly from the last electrical conductance plateau at $1 G_{0}$ and the smooth decrease of $\kappa_{\mathrm{ph}}$ shortly before the breaking of the atomic wire.

In Fig. 4 density plots of the different transport quantities are shown. Figure 4(a) displays the density plot for conductance $G$ over displacement. We find mainly three areas of increased counts centered at $1 G_{0}, 2.7 G_{0}$ and $4.5 G_{0}$, as expected from the one-dimensional histogram in Fig. 3(a). The corresponding preferred configurations for gold are bridges consisting of pentagonal rings connected by a single atom [left inset in Fig. 2(b)], structures with only two or three atoms in the narrowest part [middle inset in Fig. 2(b)], and single-atom contacts [right inset in Fig. 2(b)]. The transitions between these configurations happen relatively fast on a nanosecond time scale.

Figure 4(b) shows the total thermal conductance $\kappa$ over the displacement. As expected from the histograms in Fig. 3, the differences between $G$ and $\kappa$ are only minor and mainly stem from the small phononic contribution $\kappa_{\mathrm{ph}}$. Since all the major features of Fig. 4(a) can be recovered, we refrain from discussing them again.

Figure $4(\mathrm{c})$ plots $\kappa_{\text {ph }}$ over $G$. While the electrode displacement is a measure for the simulation time, $G$ is an approximate measure of the narrowest cross section of the contact. We find that $\kappa_{\text {ph }}$ shows a weak decrease with decreasing $G$ for large values $\left(G>6 G_{0}\right)$. The decrease becomes larger for 

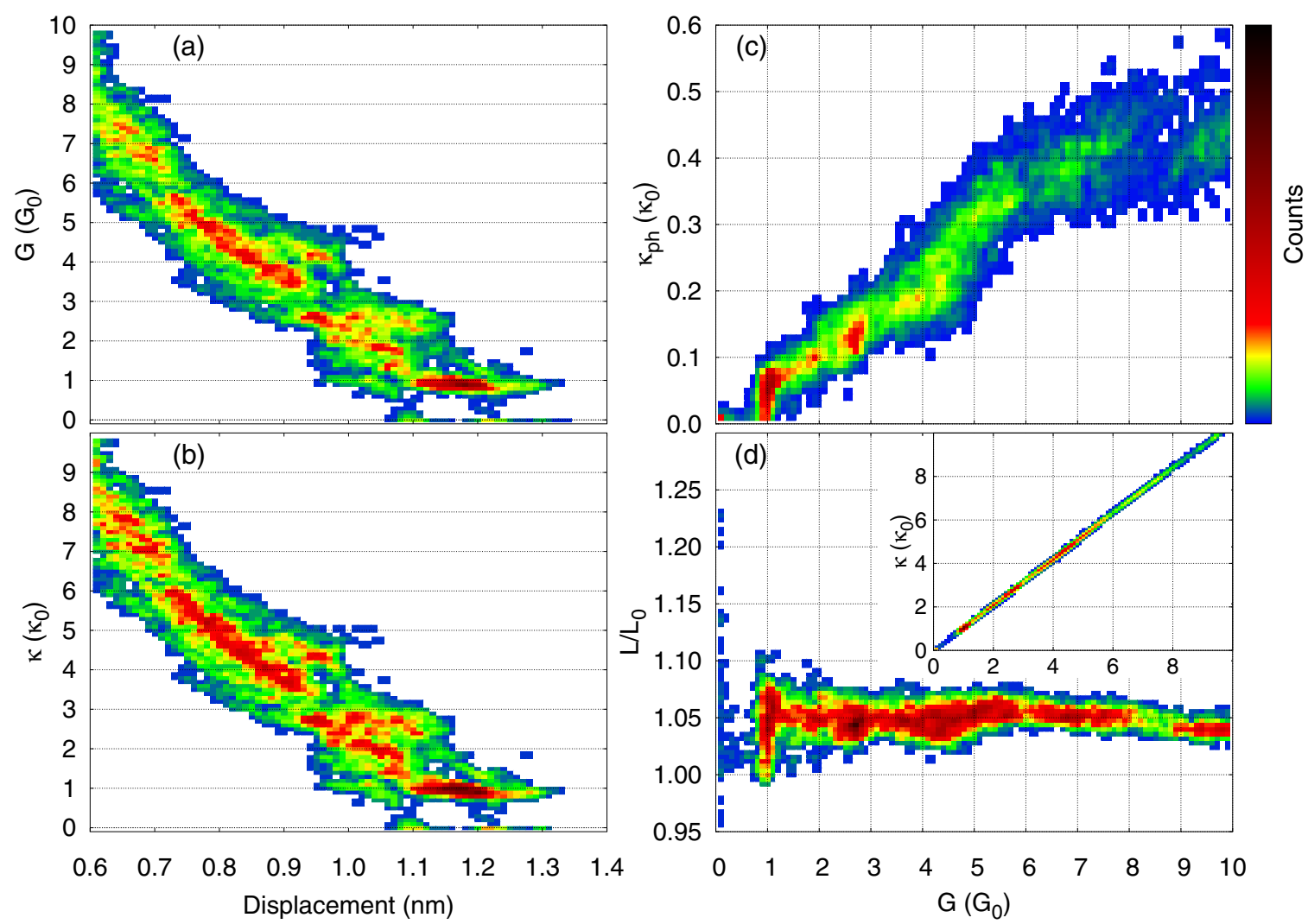

FIG. 4. Density plots for Au. (a) Electrical conductance $G$ and (b) thermal conductance $\kappa$ over electrode displacement, respectively. (c) Phononic thermal conductance $\kappa_{\mathrm{ph}}$ and (d) Lorenz ratio $L / L_{0}$ as well as in the inset thermal conductance $\kappa$ over electrical conductance $G$, respectively. For the density plots we use bin sizes of $0.1 G_{0}$ and $0.1 \kappa_{0}$ for the electrical and thermal conductances, 0.004 for the Lorenz ratio, and $0.01 \mathrm{~nm}$ for the displacement bins, corresponding to $1 \mathrm{~ns}$ at the chosen electrode separation speed.

$4 G_{0}<G<6 G_{0}$ in the range of the pentagonal ring structures. The increased slope suggests a high rigidity of these bridges, since $\kappa_{\mathrm{ph}}$ decreases faster with $G$ than for other structures. For smaller $G$, the decrease of $\kappa_{\text {ph }}$ flattens again until $\kappa_{\text {ph }}$ drops to zero rapidly at $1 G_{0}$.

In Fig. 4(d) the density plot of the Lorenz ratio $L / L_{0}$ is depicted. For the largest structures at $10 G_{0}$ it is centered around 1.04 and increases slightly to 1.05 for smaller structures between $1 G_{0}$ and $6 G_{0}$. The variation of $L / L_{0}$ increases for narrower wires, and, although still moderate with $1 \leqslant$ $L / L_{0} \leqslant 1.1$, in the contact regime it is largest at $1 G_{0}$. As can be seen in Fig. 2, while $G$ remains in the plateau at $1 G_{0}, \kappa_{\mathrm{ph}}$ varies from $0.1 \kappa_{0}$ to 0 , which nicely explains the range of variation of $L / L_{0}$. We have attained only few counts in the tunneling regime (corresponding roughly to the conductance regime $0 \lesssim G / G_{0} \lesssim 0.5$ ), so that these phononic properties cannot be discussed reliably. It is a regime of strong current interest [29-31], and further theoretical studies are desirable that take into account also radiative contributions to the heat transport. Finally, the inset of Fig. 4(d) shows the total thermal conductance $\kappa$ over the conductance $G$. In accordance with the results for the Lorenz ratio, $\kappa$ increases linearly with $G$ at a slope of $L / L_{0} \cdot \kappa_{0} / G_{0}$.

To summarize, since the electronic transport quantities $G$ and $\kappa_{\mathrm{el}}$ show excellent agreement with the Wiedemann-Franz law, the average deviations of $L / L_{0}$ from 1 on the order of $5 \%$ can be attributed mainly to phononic contributions. This is compatible with our conclusions in Refs. [7,21] based on a few selected geometries described by density functional theory.

\section{B. Platinum}

\section{Single stretching process}

In analogy to Fig. 2, Fig. 5 shows a single junction stretching process for platinum. The force in Fig. 5(a) is higher than for similar structures of gold, as expected, since platinum is more rigid and has a higher melting point $[28,40]$. Figure 5(b) shows the electronic and phononic transport properties $G, \kappa_{\mathrm{el}}$, and $\kappa_{\mathrm{ph}}$ as a function of electrode displacement. We observe that $G$ and $\kappa_{\text {el }}$ agree less well than for gold, signaling purely electronic deviations from the Wiedemann-Franz law. This was also observed in Refs. [7,21] and can be attributed to the stronger energy dependence of the transmission at the Fermi energy due to $d$ states. Similar to $\mathrm{Au}, \kappa_{\mathrm{ph}}$ shows a somewhat smoother behavior than $\kappa_{\mathrm{el}}$. For instance, the drop in $\kappa_{\mathrm{el}}$ from $5 \kappa_{0}$ to $3.2 \kappa_{0}$ is not matched by $\kappa_{\mathrm{ph}}$. Also the steady decrease of $\kappa_{\mathrm{el}}$ around a displacement of $0.9 \mathrm{~nm}$ is not observed in $\kappa_{\mathrm{ph}}$, which just shows a small dip but an otherwise rather constant value. Since the $d$ orbitals of platinum, which are not spherically symmetric, contribute to the electronic transport $[12,16]$, the sensitivity of electronic transport properties to geometric changes is enhanced as compared to the phononic properties, which are derived from spherically symmetric 


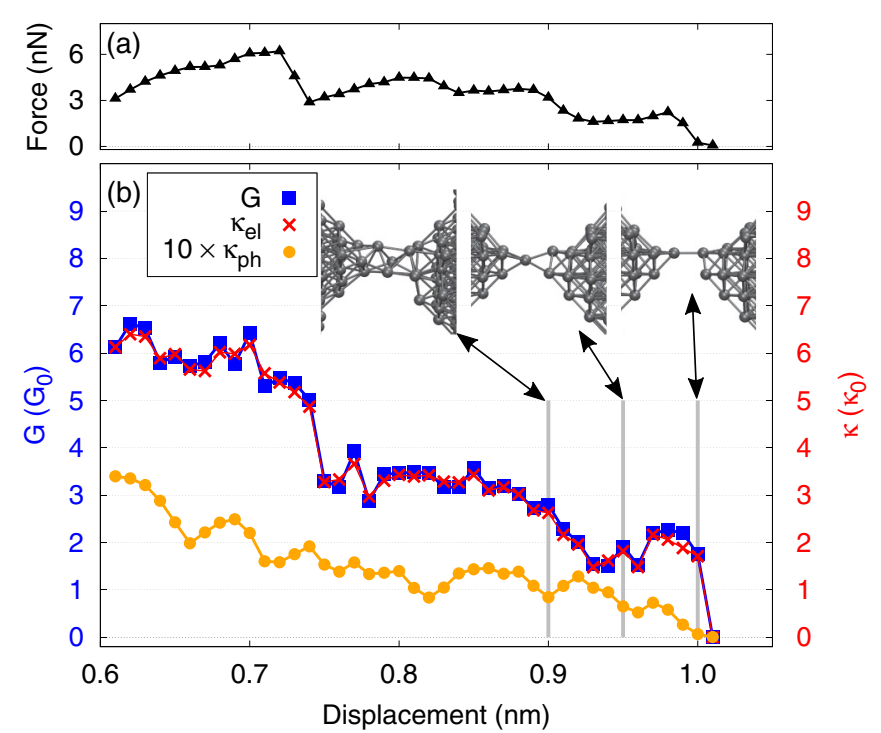

FIG. 5. Same results as Fig. 2 but for Pt. The three insets show the narrowest part of the wire at displacements of $0.90 \mathrm{~nm}, 0.95 \mathrm{~nm}$, and $1.00 \mathrm{~nm}$, as indicated by vertical gray lines.

EAM potentials. The last plateau before breaking exhibits a conductance of approximately $2 G_{0}$ as in Ref. [12]. It is higher than for gold, which is again assigned to the $d$ orbitals that contribute to electronic transport in addition to the $s$ orbitals. Similarly to gold, we observe that $\kappa_{\mathrm{ph}}$ vanishes in a rather smooth fashion that already sets in before $\kappa_{\mathrm{el}}$ drops to zero abruptly.

\section{Statistical analysis}

Now we take a look at the statistical results for platinum. Figure 6 shows the conventional conductance histograms for $G, \kappa$, and $\kappa_{\mathrm{ph}}$. The histogram for $G$ in Fig. 6(a) features regions of increased counts around $2 G_{0}, 3.6 G_{0}$, and $6.5 G_{0}$. As can be
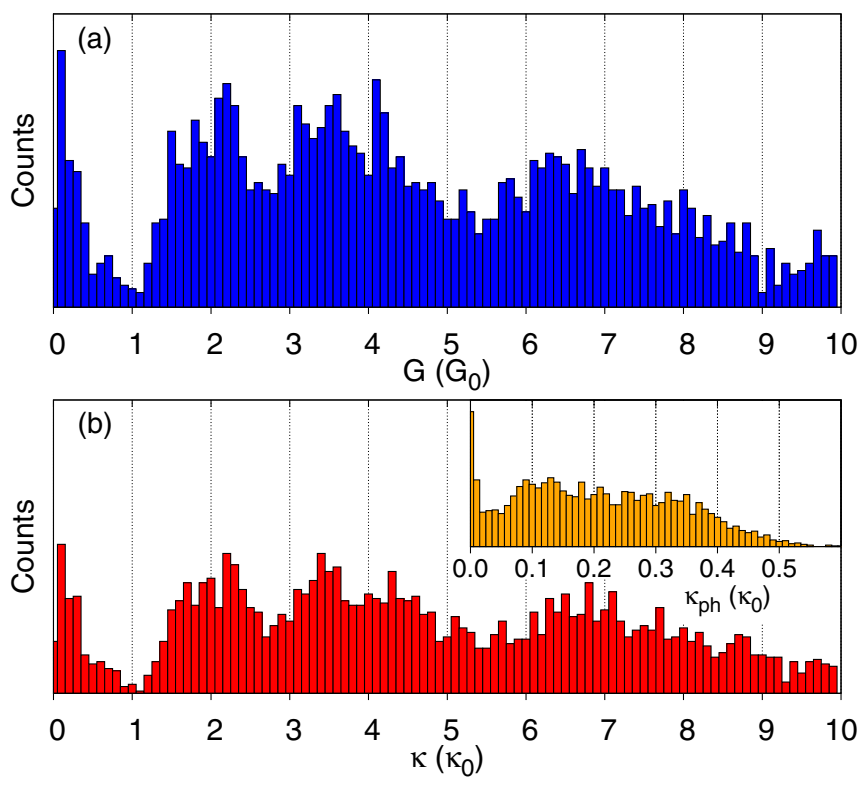

FIG. 6. Histograms as in Fig. 3 but for Pt. inferred from the single trace in Fig. 5, the region at around $2 G_{0}$ corresponds to single-atom contacts in monomer (middle inset) and dimer configuration (right inset). The second region, centered at $3.6 G_{0}$, relates to configurations with two atoms in the narrowest part, as shown in the left inset of Fig. 5, while structures at around $6.5 G_{0}$ exhibit typically four atoms at the narrowest cross section. As for $\mathrm{Au}$, the histogram of the total thermal conductance $\kappa$ in Fig. 6(b) shows a similar shape as those of $G$. The histogram of the phononic thermal conductance $\kappa_{\mathrm{ph}}$ is rather featureless.

In Fig. 7 the density plots of the various transport quantities are displayed. In Figs. 7(a) and 7(b) the conductance $G$ and the total thermal conductance $\kappa$ over the displacement show only small differences. In comparison to gold a larger spread in the transport properties at a given displacement is evident for platinum.

Figure 7 (c) shows the two-dimensional histogram of phononic thermal conductance $\kappa_{\mathrm{ph}}$ versus electrical conductance $G$. In contrast to the results for gold, $\kappa_{\mathrm{ph}}$ increases rather linearly with $G$ for all conductances, although with a wide spread.

This spread is also evident from the Lorenz ratio in Fig. 7(d). Being centered around 1.05 due to a $5 \%$ offset from 1 by phononic effects, it can vary from $0.9 \lesssim L / L_{0} \lesssim 1.2$ for the single-atom contact configurations. Opposite to Au electronic effects may contribute up to $10 \%$ to variations around the mean in $\mathrm{Pt}$, as can be seen from the electronic Lorenz ratio $L_{\mathrm{el}} / L_{0}$ [21]. Another $10 \%$ of variations comes from the phonons, adding up to a maximum of $20 \%$ difference from the Wiedemann-Franz law. The inset in Fig. 7(d) visualizes the linear relationship between $\kappa$ and $G$.

\section{Aluminum}

\section{Single stretching process}

In Fig. 8 the results of a single stretching process for an aluminum junction are plotted. Figure 8(a) shows the force to be smaller than for gold. In Fig. 8(b), the conductance $G$ and the electronic thermal conductance $\kappa_{\mathrm{el}}$ manifest a high degree of correlation, meaning that the Wiedemann-Franz law is well fulfilled. Both $G$ and $\kappa_{\mathrm{el}}$ show large fluctuations. We attribute them to the relative softness of the material compared to gold and platinum. This can be quantified by the lowest Young's modulus in the set of metals studied or the lowest melting point $[28,40]$. For this reason atomic reconfigurations occur easily at relatively small forces. As compared to $\mathrm{Au}$, $p$ orbitals contribute strongly to charge transport beside $s$ orbitals. Similar to the $d$ orbitals in platinum, the $p$ orbitals are not spherically symmetric and hence the electronic structure is quite sensitive to changes in the atomic configurations.

As previously observed for $\mathrm{Au}$ and $\mathrm{Pt}$, the phononic thermal conductance $\kappa_{\mathrm{ph}}$ shows a relatively smooth decay and no clear correlation with $\kappa_{\mathrm{el}}$. For instance, while $\kappa_{\mathrm{el}}$ decreases significantly from $10 \kappa_{0}$ to below $6 \kappa_{0}$ in a range of displacements from 0.9 to $1 \mathrm{~nm}, \kappa_{\mathrm{ph}}$ decreases to a local minimum, and rises again to a similar value as before. And around the displacement of $1.1 \mathrm{~nm} \kappa_{\mathrm{el}}$ shows two peaks, while $\kappa_{\text {ph }}$ decreases smoothly. We observe again that $\kappa_{\text {ph }}$ drops to zero faster than $\kappa_{\mathrm{el}}$, as is evident before the rupture of the contact. 

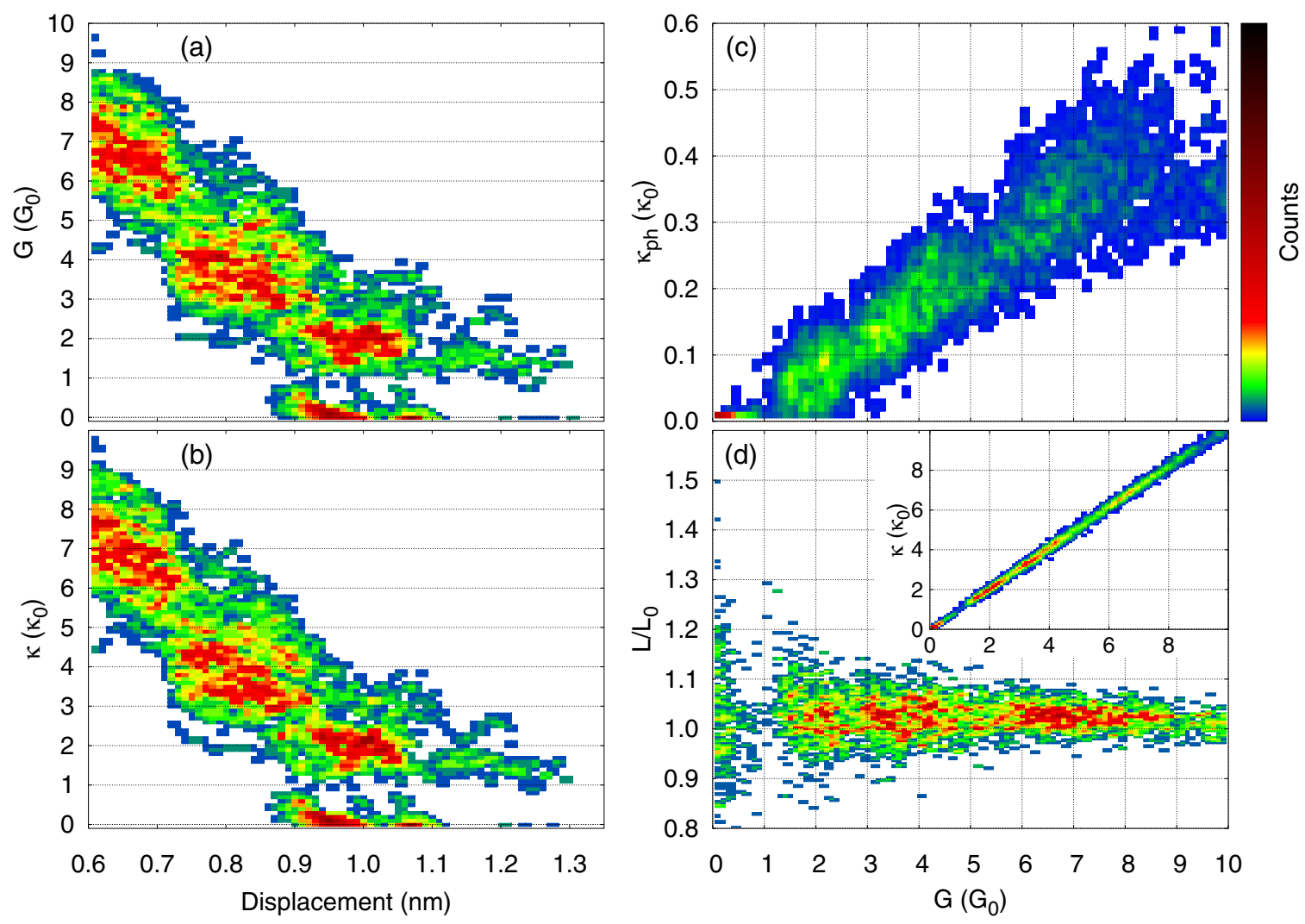

FIG. 7. Density plots as in Fig. 4 but for Pt.

The middle inset in Fig. 8(b) shows a monomer structure, which yields a conductance of $2 G_{0}$. It is realized throughout the long plateau at $2 G_{0}$, as also suggested by the leftmost geometry in the inset of Fig. 8(b) at the beginning of the plateau. We find only two data points with a conductance of
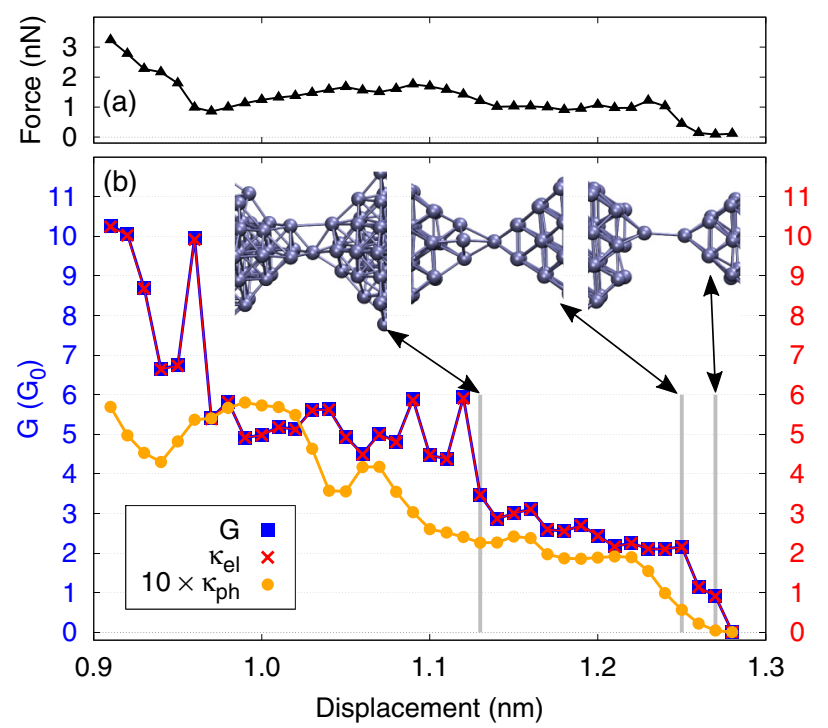

FIG. 8. Same results as in Fig. 2 but for Al. The three insets show the narrowest part of the wire at displacements of $1.13 \mathrm{~nm}, 1.25 \mathrm{~nm}$, and $1.27 \mathrm{~nm}$, as indicated by vertical gray lines. about $1 G_{0}$ for the dimer structure shown as the right inset at a displacement of $1.27 \mathrm{~nm}$.

\section{Statistical analysis}

Figure 9 depicts the histograms for the conductance $G$, the total thermal conductance $\kappa$, and the phononic heat
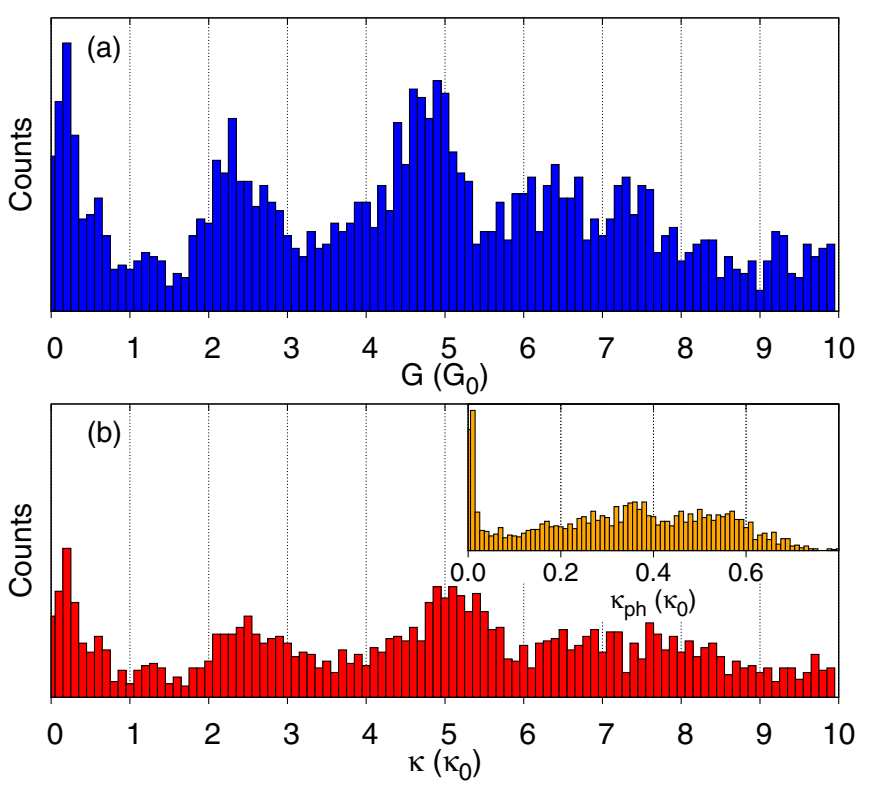

FIG. 9. Histograms as in Fig. 3 but for Al. 

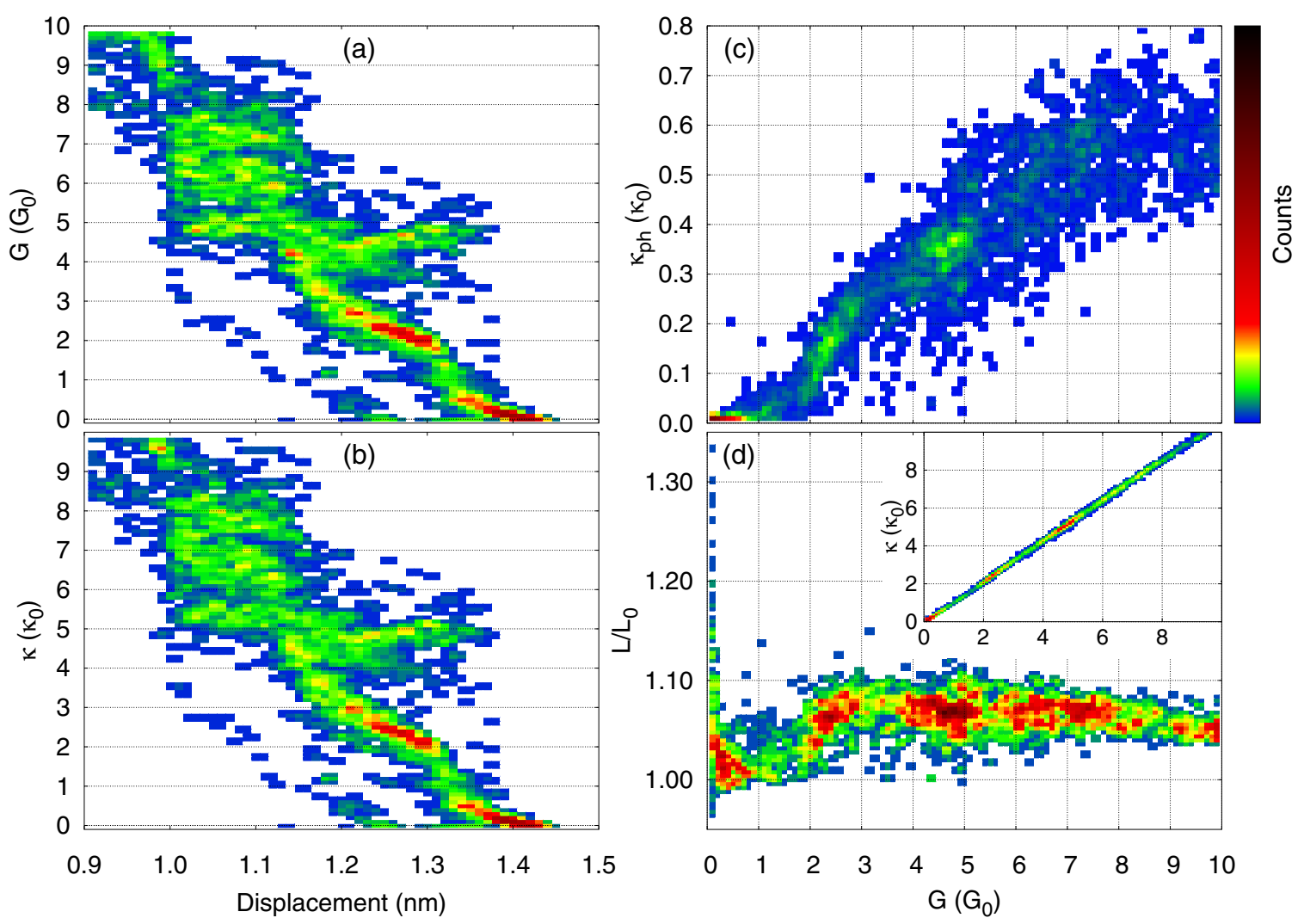

FIG. 10. Density plots as in Fig. 4 but for Al.

conductance $\kappa_{\mathrm{ph}}$. In the histogram for $G$ in Fig. 9(a) the counts centered at $1.2 G_{0}$ can be assigned to dimer configurations, as shown in the right inset of Fig. 8(b). Due to the relative instability of these dimer configurations at room temperature, the number of data points at this conductance is low. Let us note that the absence of a peak in the electrical conductance histogram around $1 G_{0}$ is at variance with experimental findings at low temperature [41]. The instability of the dimer structures in our room-temperature simulations may be a shortcoming of the chosen standard EAM potential. A larger peak at $2.3 G_{0}$ originates from monomer configurations, similar to that shown in the middle inset in Fig. 8(b). The increased count number at $4.8 G_{0}$ stems from atomic configurations with pentagonal rings and a single atom in the middle, as discussed for Au before.

The histogram for $\kappa$ in Fig. 9(b) possesses the same overall shape as the histogram for $G$. The histogram for the phononic thermal conductance $\kappa_{\mathrm{ph}}$ in the inset of Fig. 9(b) shows no distinct features but a broad distribution. The maximum of counts around $0.36 \kappa_{0}$ is related to the pentagonal ring configurations.

The analysis in terms of density plots is depicted in Fig. 10. The conductance $G$, which is related to the displacement in Fig. 10(a), shows a large variation for the different simulation runs in terms of the time point, at which contacts narrow and finally break. A large area of increased counts between displacements of 1.0 and $1.4 \mathrm{~nm}$ at a conductance of around $4.8 G_{0}$ illustrates the high probability of formation of the stable pentagonal ring configurations. It is also well visible that contacts tend to break at a large cross section and before the dimer structures with a conductance of around $1 G_{0}$ are realized.

The total thermal conductance $\kappa$ in Fig. 10(b) shows a very similar behavior as $G$ due to a small $\kappa_{\mathrm{ph}}$ and the WiedemannFranz law. We therefore do not discuss it any further.

Figure $10(\mathrm{c})$ relates $\kappa_{\mathrm{ph}}$ and $G$. An interesting feature is that $\kappa_{\text {ph }}$ stays basically below $0.1 \kappa_{0}$ for $G$ between $1 G_{0}$ and $2 G_{0}$. At roughly $2 G_{0}$ the phononic thermal conductance suddenly increases rapidly to nearly $0.2 \kappa_{0}$. This suggests a high sensitivity of $\kappa_{\mathrm{ph}}$ to reconfigurations of single-atom contacts but we can also relate this observation to the instability of dimer structures, for which the phononic transport is clearly reduced. For $G>2.5 G_{0}, \kappa_{\text {ph }}$ increases linearly.

The Lorenz ratio in Fig. 10(d) is centered between 1.00 and 1.05 for $G<2 G_{0}$ and between 1.05 and 1.08 for $G \geqslant 2 G_{0}$. In Ref. [21] we have pointed out that the phononic contribution $\kappa_{\mathrm{ph}}$ may reach $40 \%$ of $\kappa_{\mathrm{el}}$ for a dimer configuration. A main reason for this prediction is a possible suppression of the electronic transport in such junction geometries, leading to $G \approx 0.5 G_{0}$ and a correspondingly small $\kappa_{\mathrm{el}}$. We could not reproduce this finding here, as the dimer junctions were too unstable to yield a statistically significant amount of data points. The inset of Fig. 10(d) demonstrates the good linear relationship between $\kappa$ and $G$ with a slope proportional to $L / L_{0}$.

\section{CONCLUSIONS}

In this paper we have introduced a new computational scheme to study the phonon thermal conductance of 
nanojunctions. It is general and can be applied to any nanosystem, for which interatomic potentials are known. By combining the NEMD technique with a tight-binding parametrization and the Landauer-Büttiker formalism, we have illustrated its use for the description of transport properties of metallic atomic contacts by investigating the electrical conductance as well as electronic and phononic contributions to the thermal conductance. Motivated by recent experimental tests of the Wiedemann-Franz law at the atomic scale and at room temperature [7-9], our main focus was to estimate the contributions of phonons and electrons to the heat conductance. Our method proved to be computationally fast enough to perform several ten to hundred stretching simulations for atomic contacts consisting of hundreds of atoms. Detailed requirements of computational resources for the presented study are reported in Sec. A.

Concerning the validity of the Wiedemann-Franz law in metallic atomic contacts, for $\mathrm{Au}$ and $\mathrm{Pt}$ we showed that at room temperature mean deviations due to phonons are around $5 \%$, in agreement with a previous density-functionaltheory-based analysis [21] and with experimental findings [7]. Maximum deviations in the contact region were below $10 \%$ for Au mainly due to phonons but could range up to $20 \%$ for Pt. Electronic and phononic effects are responsible for this enhanced deviation in $\mathrm{Pt}$ to a similar extent. For $\mathrm{Al}$ atomic junctions we are not aware of any measurements of the thermal conductance, and our calculations should thus be seen as predictions. In this case, we found deviations from the Wiedemann-Franz law to be on average below $8 \%$ with maximum deviations up to around $10 \%$. Previous predictions of substantial deviations of up to $40 \%$ by some of the present authors [21] could not be confirmed due to an instability of dimer junction geometries at room temperature with the chosen EAM potential.

We observe a difference in the sensitivity to atomic reconfigurations between electronic and phononic thermal transport. Especially for $\mathrm{Pt}$ and $\mathrm{Al}$ contacts, the electronic thermal conductance shows more variation than the phononic thermal conductance. We attribute this to additional contributions of $d$ and $p$ orbitals to transport, respectively, which are not spherically symmetric in contrast to the EAM potentials used. We also find a rapid decrease in the phononic thermal conductance contributions before the final breaking of atomic wires, as signaled by a sharp drop in the electrical conductance. We attribute it to a weakening of the mechanical coupling within the wires that sets in already before electronic overlap is lost and indicates that an atomic contact becomes unstable.

In conclusion, the computational approach presented in this paper reproduces experimental findings for different metals and can be used to make predictions for unexplored materials. At the same time it is computationally efficient enough to facilitate a statistical analysis of charge and heat transport properties.

As an outlook, to further improve the computational results, a careful assessment of the performance of the employed EAM potentials for geometrical and vibrational properties of metallic nanostructures is desirable. Ab initio molecular dynamics could be used as a reference, and a survey of different interatomic potentials and force fields would be ideal, along the lines recently presented for bulk materials [42].
Further extensions constitute the analysis of additional starting geometries or the temperature dependence of electronic and phononic thermal conductance contributions.

\section{ACKNOWLEDGMENTS}

We thank J. Nieswand for preliminary studies during his Bachelor thesis regarding ways to determine the phonon thermal conductance with molecular-dynamics methods [43] and J. C. Klöckner for stimulating discussions on the comparison between molecular-dynamics and density-functionaltheory results for phonon heat transport. D.O.M., M.M., P.N., and F.P. were funded by the Collaborative Research Center (SFB) 767 of the German Research Foundation (DFG). F.M., M.M., and P.N. gratefully acknowledge the Gauss Centre for Supercomputing e.V. (www.gauss-centre.eu) for providing computing time through the John von Neumann Institute for Computing (NIC) on the Supercomputer JUWELS at Jülich Supercomputing Centre (JSC). An important part of the numerical modeling was furthermore carried out on the computational resources of the bwHPC program, namely the bwUniCluster and the JUSTUS HPC facility.

\section{APPENDIX: SINGLE GOLD STRETCHING PROCESS AND COMPUTATIONAL COSTS}

In this Appendix, we present a full junction stretching event for $\mathrm{Au}$. In addition, we discuss the computational costs of our simulations.

In Figs. 2, 5, and 8 we showed a part of the full stretching processes, respectively, focusing on the end before contact rupture. For completeness we display in Fig. 11 the full stretching simulation for the Au junction of Fig. 2, i.e., we extend the plot to the full range of displacements studied.

In Fig. 11 it is nicely visible that $G$ and $\kappa_{\mathrm{el}}$ follow each other closely for all separations, as expected from the Wiedemann-Franz law. It is also evident that these electronic transport properties exhibit jumps, when the force changes

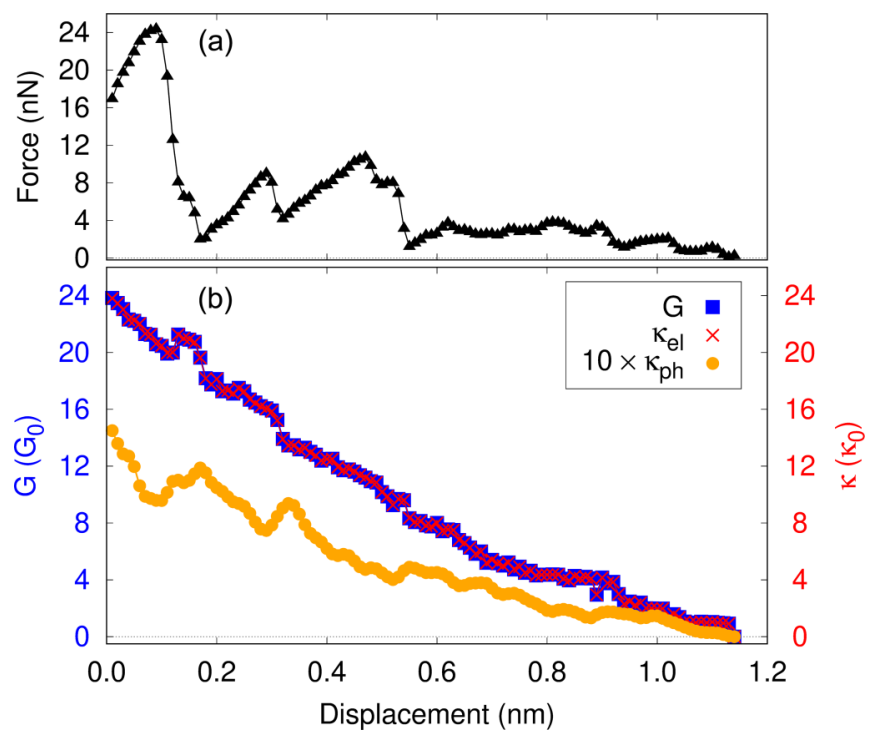

FIG. 11. The single stretching process for Au of Fig. 2, but showing the full range of displacements analyzed. 
abruptly in plastic stages and atoms rearrange. During elastic stages $G$ and $\kappa_{\text {el }}$ tend to decrease rather continuously as the nanowire narrows, but before rupture several rather horizontal conductance plateaus are found. We observe that $\kappa_{\mathrm{ph}}$ decreases more or less continuously in elastic stages. But it typically increases during plastic stages. This is particularly pronounced at the beginning of the stretching process for displacements between 0 and $0.6 \mathrm{~nm}$. An explanation for the behavior in terms of elastic constants has been given in the main text.

Let us now discuss computational requirements. As mentioned in the discussion of Fig. 2, the contact breaks at a displacement of $1.16 \mathrm{~nm}$. At the stretching speed of $0.01 \mathrm{~m} / \mathrm{s}$, a time step of $1 \mathrm{fs}$ and adding $3 \mathrm{~ns}$ or 3000000 simulation steps for the initial temperature increase and equilibrations (see Sec. II A), this corresponds to a simulation time of $119 \mathrm{~ns}$ or 119000000 necessary molecular-dynamics steps.

Since the point of rupture is not known in advance, we actually computed 203000000 molecular-dynamics steps for the simulation shown in Fig. 11. For the NEMD-related part, including generation of geometries, force determination, and phonon thermal transport calculations, we needed $146 \mathrm{core}-\mathrm{h}$ on an Intel Broadwell/Xeon E5-2680V4 architecture. The separate electronic structure simulations for 120 atomic configurations required additional 935 core-h on an Intel Sandy Bridge/Xeon E5-2670 computer.

Let us now estimate the total computing costs required for this study. Taking into account that nanowires break at different time points, we need to multiply the computing time for gold roughly by 60 , since this is the number of stretching processes analyzed. For $\mathrm{Al}$ the simulation effort is comparable to Au. Due to the relatively short lattice constant of Pt and the larger cutoff of the EAM potential as well as more iterations spent in the self-consistent loop required to achieve charge neutrality in the electronic transport simulations, the computational effort turns out to be a factor of 1.5 larger than for $\mathrm{Au}$. Overall this means that we need to multiply the computing time above by a factor of 210 , resulting in approximately 222000 core-h for the whole study.
[1] E. Pop, Energy dissipation and transport in nanoscale devices, Nano Res. 3, 147 (2010).

[2] N. Agraït, A. L. Yeyati, and J. M. van Ruitenbeek, Quantum properties of atomic-sized conductors, Phys. Rep. 377, 81 (2003).

[3] J. C. Cuevas and E. Scheer, Molecular Electronics: An Introduction to Theory and Experiment (World Scientific, Singapore, 2017).

[4] B. Ludoph and J. M. van Ruitenbeek, Thermopower of atomicsize metallic contacts, Phys. Rev. B 59, 12290 (1999).

[5] C. Evangeli, M. Matt, L. Rincón-García, F. Pauly, P. Nielaba, G. Rubio-Bollinger, J. C. Cuevas, and N. Agrait, Quantum thermopower of metallic atomic-size contacts at room temperature, Nano Lett. 15, 1006 (2015).

[6] W. Lee, K. Kim, W. Jeong, L. A. Zotti, F. Pauly, J. C. Cuevas, and P. Reddy, Heat dissipation in atomic-scale junctions, Nature (London) 498, 209 (2013).

[7] L. Cui, W. Jeong, S. Hur, M. Matt, J. C. Klöckner, F. Pauly, P. Nielaba, J. C. Cuevas, E. Meyhofer, and P. Reddy, Quantized thermal transport in single-atom junctions, Science 355, 1192 (2017).

[8] N. Mosso, U. Drechsler, F. Menges, P. Nirmalraj, S. Karg, H. Riel, and B. Gotsmann, Heat transport through atomic contacts, Nat. Nanotechnol. 12, 430 (2017).

[9] N. Mosso, A. Prasmusinto, A. Gemma, U. Drechsler, L. Novotny, and B. Gotsmann, Quantized thermal conductance in metallic heterojunctions, Appl. Phys. Lett. 114, 123102 (2019).

[10] J. K. Gimzewski and R. Möller, Transition from the tunneling regime to point contact studied using scanning tunneling microscopy, Phys. Rev. B 36, 1284 (1987).

[11] N. Agraït, J. G. Rodrigo, and S. Vieira, Conductance steps and quantization in atomic-size contacts, Phys. Rev. B 47, 12345(R) (1993).

[12] C. J. Muller, J. M. van Ruitenbeek, and L. J. de Jongh, Conductance and Supercurrent Discontinuities in Atomic-Scale Metallic Constrictions of Variable Width, Phys. Rev. Lett. 69, 140 (1992).
[13] J. M. Krans, C. J. Muller, I. K. Yanson, T. C. M. Govaert, R. Hesper, and J. M. van Ruitenbeek, One-atom point contacts, Phys. Rev. B 48, 14721 (1993).

[14] E. Scheer, N. Agraït, J. C. Cuevas, A. L. Yeyati, B. Ludoph, A. Martín-Rodero, G. R. Bollinger, J. M. van Ruitenbeek, and C. Urbina, The signature of chemical valence in the electrical conduction through a single-atom contact, Nature (London) 394, 154 (1998).

[15] M. Dreher, F. Pauly, J. Heurich, J. C. Cuevas, E. Scheer, and P. Nielaba, Structure and conductance histogram of atomic-sized Au contacts, Phys. Rev. B 72, 075435 (2005).

[16] F. Pauly, M. Dreher, J. K. Viljas, M. Häfner, J. C. Cuevas, and P. Nielaba, Theoretical analysis of the conductance histograms and structural properties of $\mathrm{Ag}, \mathrm{Pt}$, and Ni nanocontacts, Phys. Rev. B 74, 235106 (2006).

[17] F. Pauly, J. K. Viljas, M. Bürkle, M. Dreher, P. Nielaba, and J. C. Cuevas, Molecular dynamics study of the thermopower of Ag, Au, and Pt nanocontacts, Phys. Rev. B 84, 195420 (2011).

[18] P. Makk, D. Visontai, L. Oroszlány, D. Z. Manrique, S. Csonka, J. Cserti, C. Lambert, and A. Halbritter, Advanced Simulation of Conductance Histograms Validated through ChannelSensitive Experiments on Indium Nanojunctions, Phys. Rev. Lett. 107, 276801 (2011).

[19] R. Chen, M. Matt, F. Pauly, P. Nielaba, J. C. Cuevas, and D. Natelson, Shot noise variation within ensembles of gold atomic break junctions at room temperature, J. Phys.: Condens. Matter 26, 474204 (2014).

[20] R. Vardimon, M. Matt, P. Nielaba, J. C. Cuevas, and O. Tal, Orbital origin of the electrical conduction in ferromagnetic atomic-size contacts: Insights from shot noise measurements and theoretical simulations, Phys. Rev. B 93, 085439 (2016).

[21] J. C. Klöckner, M. Matt, P. Nielaba, F. Pauly, and J. C. Cuevas, Thermal conductance of metallic atomic-size contacts: Phonon transport and Wiedemann-Franz law, Phys. Rev. B 96, 205405 (2017). 
[22] R. E. Cohen, M. J. Mehl, and D. A. Papaconstantopoulos, Tightbinding total-energy method for transition and noble metals, Phys. Rev. B 50, 14694(R) (1994).

[23] M. J. Mehl and D. A. Papaconstantopoulos, Applications of a tight-binding total-energy method for transition and noble metals: Elastic constants, vacancies, and surfaces of monatomic metals, Phys. Rev. B 54, 4519 (1996).

[24] J. L. Costa-Krämer, N. García, and H. Olin, Conductance quantization histograms of gold nanowires at $4 \mathrm{~K}$, Phys. Rev. B 55, 12910 (1997).

[25] C. J. Muller, J. M. Krans, T. N. Todorov, and M. A. Reed, Quantization effects in the conductance of metallic contacts at room temperature, Phys. Rev. B 53, 1022 (1996).

[26] E. Scheer, P. Joyez, D. Esteve, C. Urbina, and M. H. Devoret, Conduction Channel Transmissions of Atomic-Size Aluminum Contacts, Phys. Rev. Lett. 78, 3535 (1997).

[27] F. Pauly, J. K. Viljas, U. Huniar, M. Häfner, S. Wohlthat, M. Bürkle, J. C. Cuevas, and G. Schön, Cluster-based densityfunctional approach to quantum transport through molecular and atomic contacts, New J. Phys. 10, 125019 (2008).

[28] N. W. Ashcroft and N. Mermin, Solid State Physics (Hartcourt, Orlando, 1976).

[29] K. Kim, B. Song, V. Fernández-Hurtado, W. Lee, W. Jeong, L. Cui, D. Thompson, J. Feist, M. T. H. Reid, F. J. García-Vidal, J. C. Cuevas, E. Meyhofer, and P. Reddy, Radiative heat transfer in the extreme near field, Nature (London) 528, 387 (2015).

[30] K. Kloppstech, N. Könne, S.-A. Biehs, A. W. Rodriguez, L. Worbes, D. Hellmann, and A. Kittel, Giant heat transfer in the crossover regime between conduction and radiation, Nat. Commun. 8, 14475 (2017).

[31] L. Cui, W. Jeong, V. Fernández-Hurtado, J. Feist, F. J. GarcíaVidal, J. C. Cuevas, E. Meyhofer, and P. Reddy, Study of radiative heat transfer in Ångström- and nanometre-sized gaps, Nat. Commun. 8, 14479 (2017).
[32] J. C. Klöckner, R. Siebler, J. C. Cuevas, and F. Pauly, Thermal conductance and thermoelectric figure of merit of $\mathrm{C}_{60}$-based single-molecule junctions: Electrons, phonons, and photons, Phys. Rev. B 95, 245404 (2017).

[33] S. Plimpton, Fast parallel algorithms for short-range molecular dynamics, J. Comput. Phys. 117, 1 (1995).

[34] LAMMPS Molecular Dynamics Simulator, http://lammps.sandia. gov.

[35] M. W. Finnis and J. E. Sinclair, A simple empirical $N$ body potential for transition metals, Philos. Mag. A 50, 45 (1984).

[36] H. W. Sheng, M. J. Kramer, A. Cadien, T. Fujita, and M. W. Chen, Highly optimized embedded-atom-method potentials for fourteen fcc metals, Phys. Rev. B 83, 134118 (2011).

[37] V. Rodrigues, T. Fuhrer, and D. Ugarte, Signature of Atomic Structure in the Quantum Conductance of Gold Nanowires, Phys. Rev. Lett. 85, 4124 (2000).

[38] J. C. Slater and G. F. Koster, Simplified LCAO method for the periodic potential problem, Phys. Rev. 94, 1498 (1954).

[39] M. Bürkle and Y. Asai, How to probe the limits of the Wiedemann-Franz law at nanoscale, Nano Lett. 18, 7358 (2018).

[40] G. W. C. Kaye and T. H. Laby, Tables of Physical and Chemical Constants and Some Mathematical Functions, 14th ed. (Longman, London, 1973).

[41] A. I. Yanson and J. M. van Ruitenbeek, Do Histograms Constitute a Proof for Conductance Quantization? Phys. Rev. Lett. 79, 2157 (1997).

[42] K. Choudhary, F. Y. P. Congo, T. Liang, C. Becker, R. G. Hennig, and F. Tavazza, Evaluation and comparison of classical interatomic potentials through a user-friendly interactive webinterface, Sci. Data 4, 160125 (2017).

[43] J. Nieswand, Wärmetransport in Goldnanostrukturen, Bachelor thesis, University of Konstanz, 2016. 